\title{
On Minimal Length Factorizations of Finite Groups
}

\author{
María Isabel González Vasco, Martin Rötteler, and Rainer Steinwandt
}

\section{CONTENTS}

1. Introduction

2. Preliminaries

3. Some Examples and Construction Techniques

4. Simple Groups

5. Conclusions and Further Research

Acknowledgments

References
2000 AMS Subject Classification: Primary 20D60; Secondary 20B05

Keywords: Logarithmic signatures, group factorizations, simple groups
Logarithmic signatures are a special type of group factorizations, introduced as basic components of certain cryptographic keys. Thus, short logarithmic signatures are of special interest. We deal with the question of finding logarithmic signatures of minimal length in finite groups. In particular, such factorizations exist for solvable, symmetric, and alternating groups.

We show how to use the known examples to derive minimal length logarithmic signatures for other groups. Namely, we prove the existence of such factorizations for several classical groups and-in parts by direct computation-for all groups of order $<175560\left(=\operatorname{ord}\left(J_{1}\right)\right.$, where $J_{1}$ is Janko's first sporadic simple group). Whether there exists a minimal length logarithmic signature for each finite group still remains an open question.

\section{INTRODUCTION}

Public Key Cryptography nourishes on hard mathematical problems which very often, but not exclusively, arise from number theory. In the early ' 80 s, several authors explored the possibility of using group theoretical problems for cryptography [Wagner and Magyarik 85, Wagner 90, Magliveras 86]. In particular owing to Magliveras et al., there are various proposals for cryptographic schemes which make use of special factorizations (socalled logarithmic signatures) of finite groups [Magliveras 86, Magliveras et al. 02]. Besides inspiring further cryptographic research [González Vasco and Steinwandt 02, González Vasco et al. 03, Birget et al. 02, Bohli et al. 02], these factorizations are interesting mathematical objects in themselves. For example, Hajós' work on Minkowski's conjecture illustrates that for abelian groups, this kind of factorization arises in the study of high-dimensional tilings (see [Stein and Szabó 94]).

Defined to be used as keys within a cryptographic scheme, the question of finding short logarithmic signatures arises naturally. The logarithmic signatures of abelian groups considered in Rédei's theorem (see, e.g., [Stein and Szabó 94]) are also examples of logarithmic signatures of minimal possible length.

(c) A K Peters, Ltd. 1058-6458/2001 $\$ 0.50$ per page Experimental Mathematics 12:1, page 1 
In this contribution, we study the existence of minimal length logarithmic signatures for several families of finite groups: In Section 2, we give the basic definitions and notations; Section 3 details a (constructive) proof of the existence of minimal logarithmic signatures for solvable groups and recalls some other known constructions. Thereafter, we discuss how to extend these results to obtain new examples. From the arguments in Section 3, one can conclude that the smallest group for which a minimal length logarithmic signature does not exist (if there should be any!) must be a simple group. Hence, we devote Section 4 to studying several families of simple groups. We start by pointing out that there are suitable factorizations for $\operatorname{PSL}_{2}(q)$ and some other classical groups. Next we prove - in part through direct computation with a computer algebra system - that all finite groups of order $<175560$ (the order of Janko's first sporadic simple group) allow for a minimal length logarithmic signature. Whether such a factorization exists for all finite groups is to the best of our knowledge still an open problem. Some suggestions for possible directions for further research conclude the paper.

\section{PRELIMINARIES}

In a series of works, Magliveras et al. [Magliveras 02, Magliveras 86, Magliveras and Memon 92, Magliveras et al. 02] explored the possibility of building symmetric and asymmetric cryptosystems using certain group factorizations. We next recall the definition of logarithmic signatures, which are one of the mainstays of their research. For the basic related notions and results see, for instance, [Cusack 00].

Definition 2.1. Let $G$ be a finite group. Next, denote by $\alpha=\left[\alpha_{1}, \ldots, \alpha_{s}\right]$ a sequence of length $s \in \mathbb{N}_{0}$ such that each $\alpha_{i}(1 \leq i \leq s)$ is itself a sequence $\alpha_{i}=\left[\alpha_{i 0}, \ldots, \alpha_{i r_{i}-1}\right]$ with $\alpha_{i j} \in G\left(0 \leq j<r_{i}\right)$ and $r_{i} \in \mathbb{N}_{0}$. Then we call $\alpha$ a logarithmic signature for $G$ if each $g \in G$ is represented uniquely as a product

$$
g=\alpha_{1 j_{1}} \cdots \alpha_{s j_{s}}
$$

with $\alpha_{i j_{i}} \in \alpha_{i}(1 \leq i \leq s)$.

We refer to the sequences $\alpha_{i}, i=1, \ldots, s$, as blocks of $\alpha$ and to the integer $\ell(\alpha):=\sum_{i=1}^{s} r_{i}$ as length of $\alpha$.

Of course, for each group $G$ there always exists a trivial logarithmic signature $\alpha:=[[g \mid g \in G]]$ consisting of a single block. Being precise, we actually obtain ord $(G)$ ! "different" logarithmic signatures in this way, as a block of a signature is an (ordered) sequence and not just a set. The distinction of whether a block is a sequence or a set is mainly motivated by the use of logarithmic signatures in the public key scheme $M S T_{1}$ [Magliveras et al. 02], and as the subsequent discussion concerns only the length of logarithmic signatures, we can w.l.o.g. ignore this distinction.

Let us look at a more interesting example of a logarithmic signature: Assume we know a subgroup chain

$$
G=G_{0}>G_{1}>\cdots>G_{s}=\left\{e_{G}\right\},
$$

where $e_{G}$ denotes the unit element in $G$. Now take $\alpha=\left[\alpha_{i} \mid i=1, \ldots, s\right]$, a sequence such that each $\alpha_{i}=\left[\alpha_{i j} \mid j=0, \ldots,\left[G_{i-1}: G_{i}\right]-1\right]$ is a complete system of left coset representatives of $G_{i-1}$ modulo $G_{i}$. It is easy to check that $\alpha$ is a logarithmic signature for $G$. Such logarithmic signatures are called exact (left) transversal. Analogously, one can use right coset representatives to derive exact (right) transversal logarithmic signatures.

In general, it is a nontrivial problem to check whether a group has logarithmic signatures of a fixed length. Luckily, we have at least a lower bound depending on the group order (first given in [González Vasco and Steinwandt 02]), which allows us to recognize the shortest ones:

Remark 2.2. Let $G$ be a finite $\operatorname{group}$ and $\operatorname{ord}(G)=$ $\prod_{j=1}^{k} p_{j}^{a_{j}}$ the prime factorization of the order of $G$ (with $p_{1}, \ldots, p_{k}$ different prime numbers). Moreover, denote by $\mathcal{B}(G):=\sum_{j=1}^{k} a_{j} p_{j}$ the sum of the prime divisors of $\operatorname{ord}(G)$ counting multiplicity. Then for each logarithmic signature $\alpha$ for $G$, we have

$$
\ell(\alpha) \geq \mathcal{B}(G) .
$$

In the remaining part of the paper, we dwell on the problem of finding logarithmic signatures $\alpha$ for which the latter inequality is tight, i. e., where $\ell(\alpha)=\mathcal{B}(G)$ holds. Trivial examples are provided by the "empty" logarithmic signature $\alpha^{\{e\}}:=$ [ ] (of length 0) for the trivial group $\{e\}$ and by the logarithmic signature $\alpha^{C_{p}}:=\left[\left[g \mid g \in C_{p}\right]\right]$ for a cyclic group $C_{p}$ of prime order $p$. In the next section, we look at some more interesting examples.

\section{SOME EXAMPLES AND CONSTRUCTION TECHNIQUES}

We start by proving that the bound $(2-1)$ is met for solvable groups (this result can also be found in [González Vasco and Steinwandt 02]). 
Proposition 3.1. Let $G$ be a finite solvable group. Then there exists a logarithmic signature $\alpha$ for $G$ of minimal length, i. e., such that $\ell(\alpha)=\mathcal{B}(G)$.

Proof: Let $G$ be a solvable group of order $\prod_{i=1}^{k} p_{i}$ with $p_{1}, \ldots, p_{k}$ not necessarily distinct prime numbers. As $G$ is solvable, it allows for a composition series

$$
\gamma: \equiv G=G_{0}>G_{1}>\cdots>G_{k}=\left\{e_{G}\right\}
$$

where each $\left[G_{i-1}: G_{i}\right]$ is prime and $\prod_{i=1}^{k}\left[G_{i-1}: G_{i}\right]=$ $\prod_{i=1}^{k} p_{i}$. Clearly, any exact transversal logarithmic signature derived from $\gamma$ has length $\sum_{i=1}^{k} p_{i}$ and is thus of minimal length.

Remark 3.2. For abelian groups, there is an alternative construction which leads to a, in general, different, minimal length logarithmic signature: Let $H$ be a cyclic group of order ord $(H)=p^{m}$ for some prime $p$, and take $a$ any generator of $H$. Then $\alpha=\left[\alpha_{1}, \ldots, \alpha_{m}\right]$ with $\alpha_{i}=\left[e_{H}, a^{p^{i-1}}, \ldots, a^{(p-1) p^{i-1}}\right](i=1, \ldots, m)$ is a logarithmic signature for $H$ of length $m \cdot p=\mathcal{B}(H)$. Now just observe that the juxtaposition of minimal length logarithmic signatures for the cyclic $p$-primary factors of an abelian group $G$ yields a minimal length logarithmic signature for $G$.

As neither the definition of logarithmic signatures nor the proof of the bound in Remark 2.2 exploits the fact that a group allows for inverse elements, it might be worth pointing out that for finite commutative monoids, minimal length factorizations do not always exist:

Example 3.3. Using a computer algebra system, one easily checks by exhaustive search that there exist no 3 element subsets $\alpha_{1}, \alpha_{2}$ of the monoid $(\mathbb{Z} / 9 \mathbb{Z}, \cdot, 1)$ such that $\alpha_{1} \cdot \alpha_{2}=\mathbb{Z} / 9 \mathbb{Z}$ holds.

Proofs of the tightness of the bound (2-1) for other families of groups often exploit peculiarities of the underlying group structure. This is the case for the symmetric and alternating groups; the existence of minimal length logarithmic signatures for the symmetric group $S_{n}$ was first proven in [González Vasco and Steinwandt 02], and the tightness of the bound for the alternating group $A_{n}$ was stated in [Magliveras 02]. Both proofs are constructive, and, moreover, in [Bohli et al. 02], there are examples of minimal length logarithmic signatures for $S_{n}$ and $A_{n}$ which are in addition tame and totally nontransversal (for the definitions of these notions, see [Magliveras et al. $02])$.
The mentioned results for symmetric and alternating groups are in essence obtained by the same technique: Given a permutation representation of a group $G$, identify a point $\mathrm{p}$ so that its stabilizer $G_{\mathrm{p}}$ can be factored through a minimal length logarithmic signature and such that there exists a complete set of representatives of $G$ modulo $G_{\mathrm{p}}$ which moves p cyclically. Actually, analogously as in Remark 3.2, the underlying idea is to factor the group into a "product of disjoint pieces" for which a minimal length logarithmic signature exists. For the case where the "disjoint pieces" are finite groups, we can formalize this idea through the concept of a knit product of groups [Michor 89] (also called Zappa-Szép product).

Definition 3.4. Let $K$ and $H$ be finite groups. Then we call two mappings $\kappa: H \times K \longrightarrow K, \hbar: K \times H \longrightarrow H$ an automorphically knitted pair of actions for $(K, H)$, provided that

1. the mapping

$$
\begin{array}{ccc}
\psi_{\kappa}: H & \longrightarrow & S_{K} \\
h & \longmapsto & \kappa_{h}(\cdot):=\kappa(h, \cdot)
\end{array}
$$

is a group homomorphism,

2. the mapping

$$
\begin{array}{ccc}
\phi_{\hbar}: \quad K & \longrightarrow & S_{H} \\
k & \longmapsto & \hbar_{k}(\cdot):=\hbar(k, \cdot)
\end{array}
$$

is a group antihomomorphism, namely $\hbar_{e_{K}}=\mathrm{id}_{H}$ and $\forall k_{1}, k_{2} \in K: \hbar_{k_{1}} \hbar_{k_{2}}=\hbar_{k_{2} k_{1}}$,

3. $\forall k_{1}, k_{2} \in K, h \in H: \kappa_{h}\left(k_{1} k_{2}\right)=\kappa_{h}\left(k_{1}\right) \kappa_{\hbar_{k_{1}}(h)}\left(k_{2}\right)$, and

4. $\forall k_{1}, k_{2} \in K, h \in H: \hbar_{k}\left(h_{1} h_{2}\right)=\hbar_{\kappa_{h_{2}}(k)}\left(h_{1}\right) \hbar_{k}\left(h_{2}\right)$, where $S_{X}$ denotes the group of permutations on the set $X$ with functional composition as group operation, i. e., for $\sigma, \tau \in S_{X}$, we have $(\sigma \tau)(x):=\sigma(\tau(x))$.

Definition 3.5. Let $K, H$ be groups, and $(\kappa, \hbar)$ an automorphically knitted pair of actions for $(K, H)$. Then the group defined over $K \times H$ with multiplication

$$
\left(k_{1}, h_{1}\right) \cdot\left(k_{2}, h_{2}\right):=\left(k_{1} \kappa_{h_{1}}\left(k_{2}\right), \hbar_{k_{2}}\left(h_{1}\right) h_{2}\right),
$$

and unit element $\left(e_{K}, e_{H}\right)$ is called knit or Zappa-Szép product of $K$ and $H$ and denoted by $K \times_{(\kappa, \hbar)} H$.

Note that $K \times\left\{e_{H}\right\}$ and $\left\{e_{K}\right\} \times H$ are subgroups of $K \times_{(\kappa, \hbar)} H$, isomorphic respectively to $K$ and $H$. Also, 
if $\psi_{\kappa} \equiv \operatorname{id}_{K}$, then $\left\{e_{K}\right\} \times H$ is a normal subgroup of $K \times_{(\kappa, \hbar)} H$, and thus we have a semidirect product (similarly, if $\phi_{\hbar} \equiv \operatorname{id}_{H}$ ). If both conditions hold, $K \times_{(\kappa, \hbar)} H$ is a direct product of $K \times\left\{e_{H}\right\}$ and $\left\{e_{K}\right\} \times H$. Actually, the Zappa-Szép product generalizes the notions of direct and semidirect product, and it is easy to see that Zappa-Szép products can be used to derive groups that allow for a minimal length logarithmic signature:

Proposition 3.6. Let $G$ be a Zappa-Szép product of two finite groups $K$ and $H$, and suppose there are logarithmic signatures $\alpha^{K}$ for $K$ and $\alpha^{H}$ for $H$ such that $l\left(\alpha^{K}\right)=$ $\mathcal{B}(K)$ and $l\left(\alpha^{H}\right)=\mathcal{B}(H)$. Then there exists a logarithmic signature $\alpha^{G}$ for $G$ with $l\left(\alpha^{G}\right)=\mathcal{B}(G)$.

Proof: Let $\alpha^{K}$ and $\alpha^{H}$ be of the form

$\alpha^{K}=\left[\alpha_{1}^{K}, \ldots, \alpha_{s}^{K}\right], \alpha_{i}^{K}=\left[\alpha_{i 0}^{K}, \ldots, \alpha_{i r_{i}-1}^{K}\right](i=1, \ldots, s)$, $\alpha^{H}=\left[\alpha_{1}^{H}, \ldots, \alpha_{t}^{H}\right], \alpha_{i}^{H}=\left[\alpha_{i 0}^{H}, \ldots, \alpha_{i l_{i}-1}^{H}\right](i=1, \ldots, t)$, where $s, t \in \mathbb{N}_{0}$. Then clearly

$$
\alpha^{G}:=\left[\alpha_{1}^{G}, \ldots, \alpha_{s}^{G}, \alpha_{s+1}^{G}, \ldots, \alpha_{s+t}^{G}\right]
$$

where

$$
\begin{array}{rll}
\alpha_{i}^{G}:=\left[\left(\alpha_{i 0}^{K}, e_{H}\right), \ldots,\left(\alpha_{i r_{i}-1}^{K}, e_{H}\right)\right] & (i=1, \ldots, s), \\
\alpha_{s+i}^{G}:=\left[\left(e_{K}, \alpha_{i 0}^{H}\right), \ldots,\left(e_{K}, \alpha_{i l_{i}-1}^{H}\right)\right] & (i=1, \ldots, t),
\end{array}
$$

is a minimal length logarithmic signature for $G$.

Therefore, the bound in Remark 2.2 is, in particular, tight for any group $G$ that is an extension of two groups $K$ and $H$ which have logarithmic signatures of minimal length - and thus for all direct and semidirect products of groups with that property. The same applies to groups which are (set theoretically) decomposable as the product of two disjoint proper subgroups meeting the bound of Remark 2.2.

Example 3.7. In the following section, we see that the groups $\mathrm{PSL}_{2}(q)$ have minimal length logarithmic signatures, and thus one concludes the tightness of $(2-1)$ for the projective mock linear groups $\mathrm{PML}_{2}(q)$ (where $q$ is an even power of an odd prime), for $\mathrm{PSL}_{2}(q)$ is a subgroup of $\mathrm{PML}_{2}(q)$ of index two. For further discussion on projective mock linear groups, we refer to [Blackburn and Huppert 82, Chapter XI] and [Abhyankar 92].

Starting again with $\operatorname{PSL}_{2}(q)$, one also verifies immediately the existence of minimal length logarithmic signatures for general linear groups $\mathrm{GL}_{2}(q)$ :
Example 3.8. $\mathrm{As}_{\mathrm{GL}_{2}}(q)$ is a cyclic extension of $\mathrm{SL}_{2}(q)$, and $\mathrm{PSL}_{2}(q)$ is $\mathrm{SL}(2, q)$ modulo its center, the bound (2$1)$ is tight for $\mathrm{GL}_{2}(q)$, too.

Similarly, one easily sees that a finite group $G$ has a minimal length logarithmic signature if it has a subnormal series whose factors do. Thus, for instance, the bound is tight for all nearly solvable groups. ${ }^{1}$ As a result, if there exists any finite group which does not have a minimal length logarithmic signature, then the smallest (w.r.t. the group order) counterexample must be a simple group. In the remaining part of this paper, we therefore focus on simple groups.

\section{SIMPLE GROUPS}

Finite simple groups are indeed complex objects of study, but neatly classified [Gorenstein et al. 98] and relatively well understood. Actually, much research has been devoted to the problem of factoring finite simple groups as a product of two proper subgroups; for a detailed survey, see [Liebeck et al. 90]. However, the factorizations we are dealing with are significantly different: They are comprised of blocks which impose a unique factorization in the sense of Definition 2.1 on the group, but the blocks are not necessarily subgroups and in fact may have no "structure" at all. Of course, when trying to construct minimal length logarithmic signatures, we can, in particular, try to make use of known factorizations of finite simple groups and to exploit them for our purposes. To illustrate this approach, in the next section, we consider factorizations of simple groups into a product of Sylow subgroups.

\subsection{Factoring into a Product of Sylow Subgroups}

In [Holt and Rowley 93], Holt and Rowley study which groups $G$ can be written as a product $P_{1} \cdots P_{k}$ where $p_{1}, \ldots, p_{k}$ are the different prime numbers dividing $\operatorname{ord}(G)$ and each $P_{i}$ is a $p_{i}$-Sylow subgroup of $G$. They show that such a factorization is possible for several types of groups, including $\mathrm{PSL}_{2}(q)$ and $\mathrm{PGL}_{3}(q)$ for any prime power $q$. As all Sylow subgroups are solvable and therefore meet the bound $(2-1)$, we conclude the tightness of $(2-1)$ for all simple groups $\operatorname{PSL}_{2}(q)(q>3)$ and for all simple groups $\mathrm{PSL}_{3}(q)$ with $q \not \equiv 1(\bmod 3)$ (and hence, $\left.\operatorname{PSL}_{3}(q) \simeq \operatorname{PGL}_{3}(q)\right) .^{2}$

\footnotetext{
${ }^{1} \mathrm{~A}$ group is nearly solvable if it admits a subnormal series so that each of its factors is a Möbius group; all Möbius groups are solvable, except from $A_{5}$.

${ }^{2}$ For $\mathrm{PSL}_{2}(q)$, the existence of minimal length logarithmic signatures is also explored in [Magliveras 02]. However, the proof given
} 


\begin{tabular}{|l|l|}
\hline$P_{2}=\quad$ & $\langle(2,15)(3,10,12,7)(4,11,21,13)(5,17)(6,8,20,9)(14,18,16,19)$, \\
& $(2,5)(3,19,8,13)(4,10,14,20)(6,21,7,16)(9,11,12,18)(15,17)$, \\
& $(2,5)(3,8)(4,16)(6,20)(7,10)(9,12)(14,21)(15,17)$, \\
& $(3,14)(4,8)(6,19)(7,13)(9,21)(10,11)(12,16)(18,20)\rangle \leq \mathrm{PSL}_{3}(4)$ \\
& $\langle(1,12,5)(2,8,7)(3,13,15)(4,10,19)(6,14,18)(9,16,17)$, \\
& $(1,5,12)(2,13,9)(3,17,7)(6,14,18)(8,15,16)$ \\
& $(11,21,20)\rangle \leq \mathrm{PSL}_{3}(4)$ \\
\hline$P_{3}=$ & $\langle(1,5,17,2,15)(3,21,4,8,6)(7,11,12,9,19)$ \\
& $(13,16,20,14,18)\rangle \leq \mathrm{PSL}_{3}(4)$ \\
\hline$P_{7}=$ & $\langle(1,20,18,10,8,12,19)(2,13,6,4,17,15,3)$ \\
& $(5,21,16,9,14,11,7)\rangle \leq \mathrm{PSL}_{3}(4)$ \\
\hline
\end{tabular}

TABLE 1. Factorization $\mathrm{PSL}_{3}(4)=P_{7} P_{3} P_{2} P_{5}$.

Also for $q \equiv 1(\bmod 3)$ such a factorization of $\operatorname{PSL}_{3}(q)$ into a product of Sylow subgroups - and thereby a logarithmic signature of minimal possible length - can be available: Representing $\mathrm{PSL}_{3}(4)$ as subgroup $\langle\alpha, \beta\rangle \leq$ $S_{21}$ with generators

$$
\begin{aligned}
\alpha= & (4,11,20)(5,15,17)(6,16,18)(7,14,13) \\
& (8,12,9)(10,21,19) \\
\beta= & (1,8,21,16,15,3,2)(4,10,20,18,17,9,7) \\
& (5,12,11,14,19,13,6),
\end{aligned}
$$

and choosing Sylow subgroups $P_{2}, P_{3}, P_{5}, P_{7}$ as given in Table 1, we can express $\mathrm{PSL}_{3}(4)$ as a product $\mathrm{PSL}_{3}(4)=$ $P_{7} P_{3} P_{2} P_{5}$. At this, as always throughout the sequel, the product $(\sigma \pi) \in S_{n}$ of two permutations $\sigma, \pi \in S_{n}$ is understood to be the permutation that maps each $i \in\{1, \ldots, n\}$ to $\pi(\sigma(i))$. The correctness of this factorization is easily checked by means of a computer algebra system like GAP [Team 97] or Magma [Bosma et al. 97].

Similar factorizations can be obtained for $\mathrm{PSU}_{4}(2)$ and $\mathrm{PSU}_{3}(4)$. Namely, representing $\mathrm{PSU}_{4}(2)$ as subgroup $\langle\gamma, \delta\rangle \leq S_{45}$ with generators

$$
\begin{aligned}
\gamma= & (2,5,3)(4,12,7)(6,17,10)(8,21,14)(9,19,13) \\
& (11,29,20)(16,30,25)(18,28,27)(22,26,32) \\
& (23,36,31)(33,35,39)(34,37,41)(40,42,43), \\
\delta= & (1,2,4,8,15,24)(3,6,11,21,31,37) \\
& (5,9,16,14,23,34)(7,13,22,33,40,20)(10,18,25) \\
& (12,17,26,35,42,30)(19,28,29)(32,38,43) \\
& (36,41,45)(39,44),
\end{aligned}
$$

and choosing Sylow subgroups $P_{2}, P_{3}, P_{5}$ as listed in Table 2, we can express $\operatorname{PSU}_{4}(2)$ as a product $\operatorname{PSU}_{4}(2)=$

there does not cover all choices of $q$. For example, for $\mathrm{PSL}_{2}(13)$, no logarithmic signature can be obtained in this way.
$P_{3} P_{2} P_{5}$. Juxtaposing minimal length logarithmic signatures for these solvable factors yields the required minimal length logarithmic signature for $\mathrm{PSU}_{4}(2)$.

Based on the representation $\operatorname{PSU}_{3}(4)=\langle\epsilon, \zeta\rangle \leq S_{65}$ with

$$
\begin{aligned}
\epsilon= & (2,9,50,12,61,38,14,3,15,4,27,63,52,23,6) \\
& (5,32,21,10,53,33,18,11,55,45,22,47,30,16,8) \\
& (7,62,39,29,51,25,60,17,43,42,49,44,28,34,36) \\
& (13,48,57,59,46,41,24,20,37,54,58,35,40,19,26) \\
& (56,64,65), \\
\zeta= & (1,2,4,8,18,39,26,48,59,44,22,21,6,15,31) \\
& (3,7,10,23,33,47,27,16,34,9,20,41,61,40,58) \\
& (5,12,17,37,55,54,60,38,32,56,28,52,63,62,64) \\
& (11,25,43,45,51,46,49,50,57,65,13,14,29,19,42) \\
& (24,35,36),
\end{aligned}
$$

in Table 3, a respective factorization $\operatorname{PSU}_{3}(4)=$ $P_{13} P_{5} P_{2} P_{3}$ of $\mathrm{PSU}_{3}(4)$ into Sylow subgroups is specified.

In general, we certainly cannot expect that a logarithmic signature of minimal length for a given finite (simple) group can be obtained by means of a factorization into Sylow subgroups. Actually, Holt and Rowley prove in [Holt and Rowley 93] that such a factorization does not exist for the simple group $\mathrm{PSU}_{3}(3)$. At the moment, we do not know whether there exists a finite simple groupor equivalently any finite group-for which no logarithmic signature at all meeting the bound $(2-1)$ exists. In the next section, we show that, in particular, for all sporadic simple Mathieu groups, a minimal length logarithmic signature exists, and thereafter, we prove that all simple groups of order $<175560$ (the order of Janko's first sporadic simple group) allow for a minimal length logarithmic signature, too. This implies that the bound 


\begin{tabular}{|c|c|c|}
\hline$P_{2}$ & & $\begin{array}{l}\langle(1,30,45,23)(2,28,3,42)(4,17,16,19)(5,11)(6,9,26,35) \\
(7,43,20,27)(8,31,41,12)(10,44,39,18)(13,40,32,38) \\
(14,36,37,29)(15,24,34,21)(22,33) \\
(1,15,17,6)(2,44,31,13)(3,32,12,18)(4,40,23,39)(5,22)(7,20) \\
(8,9,42,24)(10,30,38,16)(11,33)(14,37)(19,34,45,26) \\
(21,28,35,41)(27,29)(36,43) \\
(1,21,17,35)(2,10,31,38)(3,40,12,39)(4,32,23,18)(5,33) \\
(6,41,15,28)(7,20)(8,26,42,34)(9,19,24,45)(11,22) \\
(13,16,44,30)(14,37)(27,36)(29,43)\rangle \leq \operatorname{PSU}_{4}(2)\end{array}$ \\
\hline$P_{3}$ & $=$ & $\begin{array}{l}\langle(1,27,3,19,16,12,11,34,25)(2,32,26,17,8,21,33,24,36) \\
(4,30,22,37,43,45,10,35,31)(5,41,29) \\
(6,20,44,28,13,14,40,38,23)(7,42,18)(9,39,15) \\
(2,44,34)(3,40,24)(4,22,30)(5,42,41)(6,32,12)(7,18,39) \\
(8,25,28)(9,15,29)(10,31,35)(13,20,38)(14,27,17)(16,33,23) \\
(21,36,26)(37,45,43)\rangle \leq \mathrm{PSU}_{4}(2)\end{array}$ \\
\hline$P_{5}$ & & $\begin{array}{l}\langle(1,30,40,34,20)(2,42,21,18,37)(3,23,10,13,7)(4,35,15,27,17) \\
(5,11,33,22,25)(6,14,45,28,9)(8,38,26,36,19)(12,16,24,32,43) \\
(29,31,41,39,44)\rangle \leq \mathrm{PSU}_{4}(2)\end{array}$ \\
\hline
\end{tabular}

TABLE 2. Factorization $\mathrm{PSU}_{4}(2)=P_{3} P_{2} P_{5}$.

(2-1) must be tight for arbitrary (not necessarily simple) groups of order $<175560$.

\subsection{Mathieu Groups}

Among the sporadic simple groups, the five Mathieu groups $M_{11}, M_{12}, M_{22}, M_{23}$, and $M_{24}$ were constructed two centuries ago. They are highly transitive permutation groups and can be obtained as the automorphism groups of Steiner systems. More precisely, $M_{12}$ is the sharply 5-transitive automorphism group of a Steiner system $S(5,6 ; 12)$ and $M_{24}$ is the 5 -transitive automorphism group of a Steiner system $S(5,8 ; 24)$. We refer to [Beth et al. 99] for notations and the realization of the Mathieu groups as automorphism groups of Steiner systems. In the following, we employ a more direct construction which allows us to obtain the Mathieu groups as symmetries of the projective geometries $\mathrm{PG}\left(2, \mathbb{F}_{11}\right)$ and $\mathrm{PG}\left(2, \mathbb{F}_{23}\right)$, respectively.

We first outline an elementary construction of the Mathieu groups $M_{11}$ and $M_{12}$ based on the projective geometry PG $\left(2, \mathbb{F}_{11}\right)$. Let denote the points of the standard coordinatization of $\mathrm{PG}\left(2, \mathbb{F}_{11}\right)$. Then $M_{12}$ can be obtained as a permutation group on the set $P$. Namely, consider the following mappings (see [Gorenstein 82, Chapter 2.2]) which permute the set $P$ :

$$
f: x \mapsto x+1, \quad g: x \mapsto-\frac{1}{x}, \quad h: x \mapsto 4 x^{2}-3 x^{7} .
$$

Note that by convention $\infty=1 / 0$ and $0=1 / \infty$. The elements $f$ and $g$ generate the group $\mathrm{PSL}_{2}(11)$ which is contained in $M_{12}:=\langle f, g, h\rangle$. Explicitly, $f, g$, and $h$ give rise to the following permutations $\sigma, \tau$, and $\pi$ of $P$ :

$$
\begin{aligned}
\sigma & =(0,1,2,3,4,5,6,7,8,9,10) \\
\tau & =(0, \infty)(1,10)(2,5)(3,7)(4,8)(6,9) \\
\pi & =(2,6,10,7)(3,9,4,5)
\end{aligned}
$$

Now $M_{12}$ is a simple group of order 95040 which is sharply 5 -transitive on the points $P$. Furthermore, $M_{11}$ is defined as the stabilizer of the point 0 . Hence, $\operatorname{ord}\left(M_{11}\right)=7920$ and a direct calculation shows that

$$
M_{12}=K_{12} \cdot M_{11}
$$




\begin{tabular}{|c|c|c|}
\hline & & $\begin{array}{l}\langle(1,40,29,21)(2,23,15,20)(3,49,34,12)(4,27,46,54) \\
(5,50,30,26)(6,32,47,38)(7,35,65,25)(8,41,62,13) \\
(9,44,36,52)(10,28,45,56)(11,53,59,17)(14,61,24,63) \\
(16,19,43,37)(22,60,48,31)(33,39,58,55)(42,51,64,57) \\
(1,27,24,6)(2,8,22,37)(3,51,52,33)(4,21,38,61)(5,25,45,11) \\
(7,50,53,56)(9,64,49,39)(10,59,30,35)(12,55,36,42) \\
(13,20,43,31)(14,47,29,54)(15,62,48,19)(16,60,41,23) \\
(17,28,65,26)(32,63,46,40)(34,57,44,58) \\
(1,2,14,48)(3,30,44,45)(4,16,32,13)(5,52,10,34)(6,62,54,37) \\
(7,42,17,39)(8,27,19,47)(9,28,12,50)(11,58,35,51) \\
(15,24,22,29)(20,63,60,21)(23,61,31,40)(25,57,59,33) \\
(26,36,56,49)(38,41,46,43)(53,55,65,64) \\
(1,57,14,33)(2,35,48,11)(3,6,44,54)(4,12,32,9)(5,37,10,62) \\
(7,31,17,23)(8,30,19,45)(13,26,16,56)(15,25,22,59) \\
(20,65,60,53)(21,42,63,39)(24,58,29,51)(27,34,47,52) \\
(28,41,50,43)(36,46,49,38)(40,64,61,55)\rangle \leq \mathrm{PSU}_{3}(4)\end{array}$ \\
\hline$P_{3}$ & $=$ & $\begin{array}{l}\langle(1,21,22)(2,14,40)(3,62,42)(4,65,49)(5,41,25)(6,33,56) \\
(7,9,32)(8,55,44)(10,13,11)(12,38,53)(15,29,61)(16,59,30) \\
(17,36,46)(19,39,34)(23,31,60)(24,63,48)(26,47,51) \\
(27,57,28)(35,45,43)(37,64,52)(50,54,58)\rangle \leq \mathrm{PSU}_{3}(4)\end{array}$ \\
\hline$P_{5}$ & $=$ & $\begin{array}{l}\langle(1,40,52,42,30)(2,51,60,38,8)(3,55,10,24,63) \\
(4,37,22,33,23)(5,29,21,44,64)(6,53,43,28,9)(7,13,26,49,27) \\
(12,54,65,41,50)(14,61,34,39,45)(15,57,31,32,62) \\
(16,56,36,47,17)(19,48,58,20,46) \\
(1,47,54,5,15)(2,51,60,38,8)(3,53,49,34,22)(4,24,9,13,14) \\
(6,26,61,37,63)(7,45,23,10,28)(11,59,25,35,18) \\
(12,64,62,30,36)(16,41,21,31,52)(17,65,29,57,40) \\
(19,58,46,48,20)(27,39,33,55,43) \\
(32,42,56,50,44)\rangle \leq \mathrm{PSU}_{3}(4)\end{array}$ \\
\hline$P_{13}$ & $=$ & $\begin{array}{l}\langle(1,3,48,30,53,6,58,18,60,56,16,55,38) \\
(2,65,21,61,13,46,45,19,10,59,42,8,64) \\
(4,54,14,50,27,44,26,15,22,34,25,29,32) \\
(5,47,35,28,39,51,31,37,36,9,62,33,20) \\
(7,49,24,43,40,57,23,63,17,52,12,41,11)\rangle \leq \mathrm{PSU}_{3}(4)\end{array}$ \\
\hline
\end{tabular}

TABLE 3. Factorization $\operatorname{PSU}_{3}(4)=P_{13} P_{5} P_{2} P_{3}$.

where $K_{12} \leq M_{12}$ is the subgroup of order 12 generated by

$$
\begin{aligned}
& \alpha:=(0,7)(1,8)(2,6)(3, \infty)(4,10)(5,9), \\
& \beta:=(0,1)(2,9)(3,4)(5,6)(7,8)(10, \infty) .
\end{aligned}
$$

In terms of the generators $\sigma, \tau$, and $\pi$, we obtain the factorizations $\alpha=\pi \sigma^{2} \tau \pi^{-1} \tau \pi \tau^{-1} \sigma^{-2} \pi^{-1}$ and $\beta=$ $\pi^{-1} \tau \sigma^{2} \tau^{-1} \sigma \pi$.

Since $K_{12}$ is an abelian group isomorphic to a product $C_{2} \times C_{6}$ of two cyclic groups, we obtain from Equation (4-1) that $M_{12}$ has a logarithmic signature of minimal length if we can find one for $M_{11}$. To put it another way, we have that $M_{12}$ is a Zappa-Szép product of $K_{12}$ and $M_{11}$ (see Section 3).

Focusing on $M_{11}$, we consider the stabilizer of the point 1 and obtain a group $M_{10}$ of order 720 which is known to have a composition series $\left\{e_{M_{10}}\right\}<A_{6}<M_{10}$. Since $A_{6}$ allows for a logarithmic signature meeting the bound (2-1) [Magliveras 02, González Vasco and Steinwandt 02] and the factor group is isomorphic to $C_{2}$, we obtain that $M_{10}$ has a logarithmic signature of minimal length, and because of $M_{11}$ being isomorphic to a ZappaSzép product

$$
M_{11}=C_{11} \cdot M_{10},
$$

we can also construct minimal length logarithmic signatures for the small Mathieu groups $M_{11}$ and $M_{12}$.

4.2.1 The Mathieu groups $M_{22}, M_{23}$, and $M_{24}$. Similarly to the construction of $M_{12}$, one may obtain $M_{24}$ as a permutation group acting on the projective geometry $\mathrm{PG}\left(2, \mathbb{F}_{23}\right)$. This time, we start from $\mathrm{PSL}_{2}(23)$ which 


\begin{tabular}{|c|c|c|}
\hline group & order & possible minimal length factorization \\
\hline \hline$C_{p}(p$ prime $)$ & $p$ & trivial: $\left[\left[g \mid g \in C_{p}\right]\right]$ \\
\hline$A_{n}(n \geq 5)$ & $n ! / 2$ & $\begin{array}{c}\text { stabilizer chain (see [Magliveras 02] } \\
\text { and [Bohli et al. 02]) }\end{array}$ \\
\hline PSL $_{2}(q)(q>3)$ & $\frac{q \cdot\left(q^{2}-1\right)}{\text { gcd }(2, q-1)}$ & product of Sylow subgroups (Section 4.1) \\
\hline PSL $_{3}(q)\left(q \neq q_{3} 1\right)$ & $q^{3}\left(q^{3}-1\right)\left(q^{2}-1\right)$ & product of Sylow subgroups (Section 4.1) \\
\hline $\mathrm{PSL}_{3}(4)$ & 20160 & product of Sylow subgroups (Table 1) \\
\hline $\mathrm{PSU}_{4}(2)$ & 25920 & product of Sylow subgroups (Table 2$)$ \\
\hline $\mathrm{PSU}_{3}(4)$ & 62400 & product of Sylow subgroups $($ Table 3$)$ \\
\hline$M_{11}$ & 7920 & product of subgroups: $C_{11} \cdot A_{6} \cdot C_{2}$ \\
\hline$M_{12}$ & 95040 & product of subgroups: $\left(C_{2} \times C_{6}\right) \cdot M_{11}$ \\
\hline$M_{22}$ & 443520 & product of subgroups: PSL ${ }_{3}(4) \cdot C_{2} \cdot C_{11}$ \\
\hline$M_{23}$ & 10200960 & product of subgroups: $C_{23} \cdot M_{22}$ \\
\hline$M_{24}$ & 244823040 & product of subgroups: $S_{4} \cdot M_{23}$ \\
\hline
\end{tabular}

TABLE 4. Simple groups meeting the bound in Remark 2.2.

is generated by $f: x \mapsto x+1$ and $g: x \mapsto-\frac{1}{x}$. The mapping, (see [Gorenstein 82])

$$
h: x \mapsto-3 x^{15}+4 x^{4},
$$

defines a permutation of the points of $\mathrm{PG}\left(2, \mathbb{F}_{23}\right)$. The Mathieu group $M_{24}$ is defined as $M_{24}:=\langle\sigma, \tau, \pi\rangle$, where the permutations $\sigma, \tau$, and $\pi$ are obtained from $f, g$, and $h$. Explicitly, we have

$$
\begin{aligned}
& \sigma=(0,1,2,3,4,5,6,7,8,9,10,11,12,13,14,15, \\
& 16,17,18,19,20,21,22) \text {, } \\
& \tau=(0, \infty)(1,22)(2,11)(3,15)(4,17)(5,9)(6,19) \\
& (7,13)(8,20)(10,16)(12,21)(14,18) \text {, } \\
& \pi=(2,16,9,6,8)(3,12,13,18,4)(7,17,10,11,22) \\
& (14,19,21,20,15) \text {. }
\end{aligned}
$$

Note that $M_{24}$ can be written as a Zappa-Szép product in the following way:

$$
M_{24}=S_{4} \cdot M_{23},
$$

where $M_{23}$ denotes the stabilizer of the point 0 in $M_{24}$. The symmetric group $S_{4}:=\langle\alpha, \beta\rangle \leq M_{24}$ is generated by the permutations

$$
\begin{aligned}
\alpha:= & (0,2)(1,14)(3,8)(4, \infty)(5,20)(6,17)(7,10) \\
& (9,21)(11,18)(12,16)(13,15)(19,22) \\
\beta:= & (0,1, \infty, 12)(2,7,3,9)(4,18,13,22)(5,10,16,19) \\
& (6,11,14,21)(8,20,15,17) .
\end{aligned}
$$

We can descend one more step to $M_{22}$ since the corresponding transversal can be chosen to be a cyclic group of order 23. More precisely, we have $M_{23}=C_{23} \cdot M_{22}$, where $M_{22}$ stabilizes the points 0 and 1 , and $C_{23}$ is a cyclic group of order 23. In fact, as generator of $C_{23}$, we can choose an arbitrary element of order 23 in $M_{23}$.

Finally, $M_{22}$ can be realized as follows: We define the subgroups

$$
\begin{aligned}
C_{11}:= & \langle(2,19,9,7,18,12, \infty, 5,11,16,21) \\
& (3,17,8,10,13,20,22,14,6,4,15)\rangle \leq M_{22}
\end{aligned}
$$

and

$$
\begin{gathered}
C_{2}:=\langle(2,10)(3,19)(4,11)(7,13)(8,21)(9,15)(12,14) \\
(16,17)\rangle \leq M_{22} .
\end{gathered}
$$

Then the subset $A:=C_{2} \cdot C_{11}$ of $M_{22}$ has the property that for each point $x \in\{2, \ldots, 22, \infty\}$, there is an element $\rho \in A$ such that $\rho(2)=x$. On the other hand, the stabilizer of the points 0,1 , and 2 inside $M_{24}$ is isomorphic to the simple group $\mathrm{PSL}_{3}(4)$ of order 20160 . Thus, using the result that $\mathrm{PSL}_{3}(4)$ has a logarithmic signature of minimal possible length (see Table 1), we also obtain a logarithmic signature for $M_{22}=\mathrm{PSL}_{3}(4) \cdot A$ that meets the bound (2-1). Hence, by the previous arguments, $M_{23}$ and $M_{24}$ also have logarithmic signatures of minimal possible length.

\subsection{A Lower Bound for the Size of a Counterexample}

In Table 4, the simple groups for which we already know that the bound in Remark 2.2 is tight are listed.

In particular, this list covers all simple groups of order $\leq 2^{17}$ up to the following three exceptions:

- $\mathrm{PSU}_{3}(3)$ (of order 6048 ) 


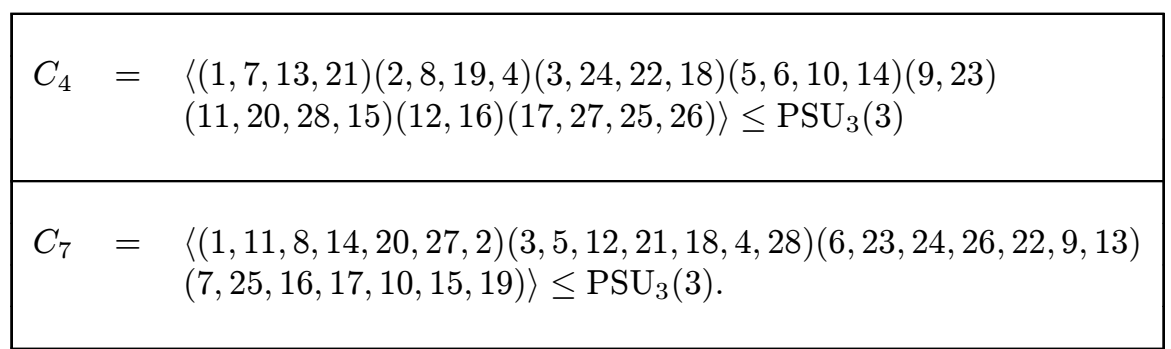

TABLE 5. Factors $C_{4}$ and $C_{7}$ of the factorization $\operatorname{PSU}_{3}(3)=G_{1} C_{4} C_{7}$.

\begin{tabular}{|c|c|c|}
\hline$C_{5}$ & $=$ & $\begin{array}{l}\langle(1,38,43,40,64)(2,50,32,63,44)(3,18,10,65,61) \\
(4,7,15,24,12)(5,19,33,54,17)(6,8,36,27,13) \\
(9,31,48,47,30)(11,49,51,20,23)(14,60,16,53,57) \\
(21,39,56,35,37)(22,46,58,45,59)(25,34,62,26,41) \\
(28,55,29,42,52)\rangle \leq \mathrm{Sz}(8)\end{array}$ \\
\hline$C_{13}$ & $=$ & $\begin{array}{l}\langle(1,46,21,18,47,51,22,31,15,4,39,6,41) \\
(2,56,32,27,5,49,3,20,40,54,50,59,23) \\
(7,42,48,61,37,65,53,36,19,8,17,45,43) \\
(9,52,55,29,38,12,62,25,57,24,11,58,30) \\
(10,63,26,14,44,35,13,34,33,16,64,28,60)\rangle \leq \mathrm{Sz}(8)\end{array}$ \\
\hline
\end{tabular}

TABLE 6. Factors $C_{5}$ and $C_{13}$ of the factorization $\mathrm{Sz}(8)=G_{1} C_{5} C_{13}$.

- $\mathrm{Sz}(8)$ (of order 29120 )

- $\mathrm{PSU}_{3}(5)$ (of order 126000 )

Subsequently, we give a logarithmic signature for each of these three groups. They have been found and verified by means of the computer algebra system Magma.

4.3.1 $\mathbf{P S U}_{3}(3)$. We represent $\mathrm{PSU}_{3}(3)$ as a subgroup $\langle\alpha, \beta\rangle \leq S_{28}$ with generators

$$
\begin{aligned}
\alpha= & (2,7,23,26,17,13,6,3)(4,19,11,28,25,24,16,9) \\
& (5,18,10,14,15,8,20,12)(21,27) \\
\beta= & (1,2,4,10,8,16,13,22)(3,7,9,17,6,12,21,5) \\
& (11,19,26,14,15,23,24,25)(27,28) .
\end{aligned}
$$

Then $\mathrm{PSU}_{3}(3)$ can be factored into a product $\langle\alpha, \beta\rangle=$ $G_{1} C_{4} C_{7}$ where $G_{1}$ is the stabilizer of 1 , and $C_{4}$, respectively, $C_{7}$, is a cyclic group of order 4 , respectively, 7 . Precise choices for $C_{4}$ and $C_{7}$ are given in Table 5 .

As the stabilizer $G_{1}$ (of order $216=\operatorname{ord}\left(\mathrm{PSU}_{3}(3)\right) /(4$. $7)$ ) is solvable, we immediately obtain a minimal length logarithmic signature for $\mathrm{PSU}_{3}(3)$ by juxtaposing minimal length logarithmic signatures for the three subgroups $G_{1}, C_{4}$, and $C_{7}$.
4.3.2 $\mathrm{Sz}(8)$. We represent the Suzuki group $\mathrm{Sz}(8)$ as subgroup $\langle\alpha, \beta\rangle \leq S_{65}$ with generators

$$
\begin{aligned}
\alpha= & (1,2)(3,4)(5,7)(6,9)(8,12)(10,13)(11,15)(14,19) \\
& (16,21)(17,23)(18,25)(20,28)(22,31)(24,33) \\
& (26,35)(27,32)(29,37)(30,39)(34,43)(36,46) \\
& (38,48)(41,51)(42,44)(45,55)(47,50)(49,58) \\
& (52,60)(53,61)(54,59)(56,62)(57,63)(64,65), \\
\beta= & (1,3,5,8)(4,6,10,14)(7,11,16,22)(9,12,17,24) \\
& (13,18,26,36)(15,20,29,38)(19,27,31,28) \\
& (21,30,40,50)(23,32,41,52)(25,34,44,54) \\
& (33,42,53,43)(35,45,56,63)(37,47,51,46) \\
& (39,49,59,60)(48,57,55,58)(61,64,62,65) .
\end{aligned}
$$

Then $\mathrm{Sz}(8)$ can be factored into a product $\mathrm{Sz}(8)=$ $G_{1} C_{5} C_{13}$ where $G_{1}$ is the stabilizer of 1 , and $C_{5}$, respectively, $C_{13}$, is cyclic of order 5 , respectively, 13 . Concrete choices for the cyclic groups $C_{5}$ and $C_{13}$ are given in Table 6 .

As the stabilizer $G_{1}$ (of order $448=\operatorname{ord}(\mathrm{Sz}(8)) /(5 \cdot 13)$ ) is solvable, we immediately obtain the required minimal length logarithmic signature for $\mathrm{Sz}(8)$ by juxtaposing minimal length logarithmic signatures for the three factors $G_{1}, C_{5}$, and $C_{13}$. 


\begin{tabular}{|c|c|c|}
\hline$C_{2}$ & $=$ & $\begin{array}{l}(1,60)(3,6)(4,27)(5,21)(7,94)(8,59)(9,12)(10,83)(11,42) \\
(13,30)(14,84)(15,58)(16,47)(17,32)(18,74)(19,115)(20,77) \\
(22,54)(23,78)(24,120)(25,82)(26,43)(28,114)(29,100)(31,76) \\
(33,106)(34,35)(36,45)(37,80)(38,39)(40,105)(41,98)(44,55) \\
(46,67)(48,79)(49,116)(50,61)(52,124)(53,71)(56,91)(57,70) \\
(62,97)(63,119)(64,92)(65,88)(66,111)(68,103)(69,109) \\
(72,96)(73,101)(75,126)(81,122)(86,107)(87,113)(90,110) \\
(93,108)(95,125)(102,121)(104,112)(118,123)\rangle \leq \mathrm{PSU}_{3}(5) \\
\end{array}$ \\
\hline$C_{3}^{(1)}$ & $=$ & $\begin{array}{l}\langle(1,81,13)(2,119,26)(3,16,105)(4,121,97)(5,120,109) \\
(6,24,113)(7,10,28)(8,40,74)(9,66,94)(11,39,49)(12,112,62) \\
(14,60,83)(15,126,122)(17,123,56)(18,76,33)(19,69,91) \\
(20,101,45)(21,87,61)(22,78,104)(23,100,65)(25,43,72) \\
(27,67,75)(29,58,36)(30,51,38)(31,118,108)(32,59,89) \\
(34,41,55)(35,82,71)(37,103,110)(42,63,86)(44,114,107) \\
(46,48,116)(47,85,115)(50,90,80)(52,77,70)(53,124,111) \\
(54,98,102)(57,84,117)(64,79,88)(68,93,106)(73,95,99) \\
(92,125,96)\rangle \leq \mathrm{PSU}_{3}(5) \\
\end{array}$ \\
\hline$C_{3}^{(2)}$ & $=$ & $\begin{array}{l}\langle(1,8,6)(2,57,56)(3,88,104)(4,79,9)(5,22,97)(7,30,67) \\
(10,58,54)(11,28,27)(12,75,106)(13,19,94)(14,50,51) \\
(15,68,66)(16,34,44)(17,36,73)(18,25,32)(20,93,112) \\
(21,26,70)(23,87,24)(29,64,111)(31,47,116)(33,105,91) \\
(35,98,84)(37,72,121)(38,83,40)(39,59,78)(41,110,71) \\
(42,43,69)(45,108,123)(46,95,102)(48,100,77)(49,115,55) \\
(52,80,63)(53,125,96)(60,82,89)(61,124,76)(62,113,74) \\
(65,126,117)(81,114,118)(85,90,119)(86,120,92) \\
(99,109,101)(103,107,122)\rangle \leq \mathrm{PSU}_{3}(5) \\
\end{array}$ \\
\hline$C_{7}$ & $=$ & $\begin{array}{l}\langle(1,108,125,9,17,95,64)(2,39,63,34,111,25,60) \\
(3,33,68,30,74,47,107)(4,5,32,52,67,57,62) \\
(6,23,78,28,80,46,122)(7,24,50,100,48,104,15) \\
(8,18,85,93,44,51,16)(10,116,113,22,90,126,65) \\
(11,55,72,26,42,124,14)(12,77,121,75,120,84,59) \\
(13,92,56,88,118,94,73)(19,36,45,103,35,21,54) \\
(20,98,110,69,82,29,87)(27,112,109,70,117,97,89) \\
(31,99,96,79,66,81,123)(37,61,91,101,71,102,58) \\
(38,115,105,106,40,114,76)(41,119,53,49,43,86,83)\rangle \\
\end{array}$ \\
\hline
\end{tabular}

TABLE 7. Cyclic factors of the factorization $\operatorname{PSU}_{3}(5)=C_{7} C_{3}^{(1)} C_{3}^{(2)} C_{2} G_{1}$.

4.3.3 $\mathrm{PSU}_{3}(5)$. We represent $\mathrm{PSU}_{3}(5)$ as a subgroup $\langle\alpha, \beta\rangle \leq S_{126}$ with generators

$\alpha=(1,2,4,10,25)(3,7,17,41,19)(5,13,33,68,111)$

$(6,14,36,34,70)(8,20,47,81,48)(9,22,32,61,106)$

$(11,28,37,40,82)(12,30,24,55,65)$

$(15,38,77,29,64)(16,39,43,85,73)$

$(18,44,75,118,109)(21,49,93,126,120)$

$(23,53,42,83,52)(26,58,104,122,101)$

$(27,60,105,110,124)(31,35,72,116,66)$

$(45,87,123,108,96)(46,89,95,100,59)$

$(50,94,57,76,103)(51,97,114,71,107)$
$(54,88,74,63,98)(56,102,117,99,121)$

$(62,86,78,90,80)(67,79,112,92,125)$

$(69,113,84,115,119)$,

$\beta=(2,13,112,108,24,93,45,7)$

$(3,32,53,102,54,65,75,16)$

$(4,41,52,104,11,68,99,30)$

$(5,81,122,18,56,34,19,63)$

$(6,17,72,69,101,10,55,31)$

$(8,29,100,40,84,67,37,57)$

$(9,36,98,124,27,94,117,66)$

$(12,120,42,43,33,15,26,106)$ 


$$
\begin{aligned}
& (14,76,64,85,86,96,70,97) \\
& (20,73,82,83,125,50,39,113) \\
& (21,74,87,23,59,105,110,61) \\
& (22,60,88,123,78,62,90,116) \\
& (28,118,77,79,44,46,115,121) \\
& (35,80,109,48,95,49,114,126) \\
& (38,91,47,92,51,107,71,119) \\
& (58,103,89,111) .
\end{aligned}
$$

Then $\operatorname{PSU}_{3}(5)$ can be factored into a product $\langle\alpha, \beta\rangle=$ $C_{7} C_{3}^{(1)} C_{3}^{(2)} C_{2} G_{1}$ where $G_{1}$ is the stabilizer of $1, C_{2}$ is a cyclic group of order 2 , both $C_{3}^{(1)}$ and $C_{3}^{(2)}$ are cyclic groups of order 3 , and $C_{7}$ is a cyclic group of order 7. Precise choices for the four cyclic factors are given in Table 7. As the stabilizer $G_{1}$ (of order $1000=$ $\left.\operatorname{ord}\left(\mathrm{PSU}_{3}(5)\right) /(2 \cdot 3 \cdot 3 \cdot 7)\right)$ is solvable, we immediately obtain a minimal length logarithmic signature for $\operatorname{PSU}_{3}(5)$ by juxtaposing minimal length logarithmic signatures for the five subgroups $G_{1}, C_{2}, C_{3}^{(1)}, C_{3}^{(2)}$, and $C_{7}$.

Thus, if there is any finite group $G$ such that no logarithmic signature for $G$ meets the bound in Remark 2.2, then the smallest counterexample (w.r.t. the group order) has to be both simple and of order $>2^{17}$. Further on, we can exclude all simple groups covered by Table 4 , and thus the smallest group for which the tightness of $(2-1)$ is open is Janko's first sporadic group $J_{1}$ of order 175560 .

\section{CONCLUSIONS AND FURTHER RESEARCH}

Motivated by the question of finding short keys for the public key cryptosystem $M S T_{1}$, we have shown that various finite groups allow for logarithmic signatures of minimal possible length. Unfortunately, so far we could not answer the question whether there is any finite group for which the bound (2-1) is not tight, but we have shown that there can be no such group of cardinality smaller than Janko's first sporadic simple group.

Both from the mathematical and the cryptographic point of view, it would be desirable to identify further families of - not necessarily simple - groups for which the bound $(2-1)$ is tight. On the cryptographic side, the question also arises whether logarithmic signatures of minimal length can be found, where effectively factoring group elements along the logarithmic signature is computationally hard. Such logarithmic signatures would be desirable for realizing the $M S T_{1}$ public key cryptosystem.

\section{ACKNOWLEDGEMENTS}

We thank Consuelo Martínez for helpful comments and discussions. The work of the first author was partially supported by project BFM2001-3239-C03-01.

\section{REFERENCES}

[Abhyankar 92] S. S. Abhyankar. "Galois Theory on the Line in Nonzero Characteristic." Bulletin of the American Mathematical Society 27:1 (1992), 68-133.

[Beth et al. 99] Th. Beth, D. Jungnickel, and H. Lenz. Design Theory, Volume I, Second edition. Cambridge, UK: Cambridge University Press, 1999.

[Birget et al. 02] J.-C. Birget, S. S. Magliveras, and W. Wei. "Trap Doors from Subgroup Chains and Recombinant Bilateral Transversals. In Actas de la VII Reunión Española de Criptología y Seguridad de la Información; Tomo I, edited by S. González Jiménez and C. Martínez López, pp. 31-48, Oviedo: Servicio de Publicaciones, Universidad de Oviedo, 2002.

[Blackburn and Huppert 82] N. Blackburn and B. Huppert. Finite Groups III. Die Grundlehren der Mathematischen Wissenschaften. Berlin-New York: SpringerVerlag, 1982.

[Bohli et al. 02] J.-M. Bohli, M. I. González Vasco, C. Martínez, and R. Steinwandt. "Weak Keys in $M S T_{1} . "$ Cryptology ePrint Archive, Report 2002/070, 2002 .

[Bosma et al. 97] W. Bosma, J. Cannon, and C. Playoust. "The Magma Algebra System I: The User Language." Journal of Symbolic Computation 24 (1997), 235-265.

[Cusack 00] C. A. Cusack. "Group Factorizations in Cryptography." PhD thesis, University of Nebraska, 2000.

[González Vasco et al. 03] M.I. González Vasco, C. Martínez, and R. Steinwandt. "Towards a Uniform Description of Several Group Based Cryptographic Primitives." To appear in Designs, Codes and Cryptography, 2003.

[González Vasco and Steinwandt 02] M. I. González Vasco and R. Steinwandt. "Obstacles in Two Public-Key Cryptosystems Based on Group Factorizations." In Cryptology, edited by K. Nemoga and O. Grošek, pp. 23-37, Tatra Mountains Mathematical Publications, Volume 25. Bratislava: Mathematical Institute, Slovak Academy of Sciences, 2002.

[Gorenstein 82] D. Gorenstein. Finite Simple Groups. University Series in Mathematics. New York: Plenum Press, 1982.

[Gorenstein et al. 98] D. Gorenstein, R. Lyons, and R. Solomon. The Classification of the Finite Simple Groups, Mathematical Surveys and Monographs, Volume 40(1). Providence, RI: AMS 1998.

[Holt and Rowley 93] D. F. Holt and P. Rowley. "On Products of Sylow Subgroups in Finite Groups." Archiv der Mathematik 60:2 (1993), 105-107. 
[Liebeck et al. 90] M. W. Liebeck, C. E. Praeger, and J. Saxl. The Maximal Factorizations of the Finite Simple Groups and their Automorphism Groups, Memoirs of the AMS, Volume 86(432). Providence, RI: AMS, 1990.

[Magliveras 86] S. S. Magliveras. "A Cryptosystem from Logarithmic Signatures of Finite Groups." In Proceedings of the 29th Midwest Symposium on Circuits and Systems, pp. 972-975. Amsterdam: Elsevier Publishing Company, 1986.

[Magliveras 02] S. S. Magliveras. "Secret- and Public-key Cryptosystems from Group Factorizations." In Cryptology, edited by K. Nemoga and O. Grošek, pp. 11-22, Tatra Mountains Mathematical Publications, Volume 25. Bratislava: Mathematical Institute, Slovak Academy of Sciences, 2002.

[Magliveras and Memon 92] S. S. Magliveras and N. D. Memon. "Algebraic Properties of Cryptosystem PGM." Journal of Cryptology 5 (1992), 167-183.

[Magliveras et al. 02] S. S. Magliveras, D. R. Stinson, and T. van Trung. "New Approaches to Designing Public Key Cryptosystems Using One-Way Functions and Trapdoors in Finite Groups." Journal of Cryptology 15:4 (2002), 285-297.
[Michor 89] P. W. Michor. "Knit Products of Graded Lie Algebras and Groups." In Proceedings of the Winter School on Geometry and Physics, Srni 1988, Ser. II, 22, pp. 171-175, Palermo: Suppl. Rendiconti Circolo Matematico di Palermo, 1989.

[Stein and Szabó 94] S. K. Stein and S. Szabó. Algebra and Tiling. Homomorphisms in the Service of Geometry. The Carus Mathematical Monographs, No. 25. Washington, DC: The Mathematical Association of America, 1994.

[Team 97] The GAP Team. "GAP-Groups, Algorithms, and Programming." Lehrstuhl D für Mathematik, RWTH Aachen, Germany and School of Mathematical and Computational Sciences, Univ. St. Andrews, Scotland, 1997.

[Wagner 90] N. R. Wagner. "Searching for Public-Key Cryptosystems." In Proceedings of the 1984 Symposium on Security and Privacy (SSP '84), pp. 91-98. Los Alamitos, CA: IEEE Computer Society Press, 1990.

[Wagner and Magyarik 85] N. R. Wagner and M. R. Magyarik. "A Public Key Cryptosystem Based on the Word Problem." In Advances in Cryptology. Proceedings of CRYPTO 1984, pp. 19-36, edited by G. R. Blakley and D. Chaum, Lecture Notes in Computer Science 196. Berlin: Springer, 1985.

María Isabel González Vasco, Departamento de Matemáticas, Universidad de Oviedo, c/Calvo Sotelo, s/n, 33007 Oviedo, Spain (mvasco@orion.ciencias.uniovi.es)

Martin Rötteler, Centre for Applied Cryptographic Research, Department of Combinatorics and Optimization, University of Waterloo, Waterloo, Ontario, Canada, N2L 3G1 (mroetteler@cacr.math.uwaterloo.ca)

Rainer Steinwandt, Institut für Algorithmen und Kognitive Systeme, Arbeitsgruppe Computeralgebra, Prof. Dr. Th. Beth, Universität Karlsruhe, 76128 Karlsruhe, Germany (steinwan@ira.uka.de)

Received March 25, 2003; accepted April 17, 2003. 